\title{
LA METODOLOGÍA POR PROYECTOS COMO OPORTUNIDAD PARA LA INTRODUCCIÓN DE LA HISTORIA Y EL PATRIMONIO EN LAS AULAS DE EDUCACIÓN INFANTIL ${ }^{1}$
}

\author{
Elisabet Casanova Alfonsea \\ Laura Arias Ferrer \\ Alejandro Egea Vivancos \\ Universidad de Murcia
}

\begin{abstract}
RESUMEN: Las tendencias actuales de investigación animan a la incorporación de contenidos históricos desde los primeros años de escolarización para así fomentar el desarrollo incipiente de habilidades asociadas al pensamiento histórico. El patrimonio cultural se muestra como un recurso clave para ello, y el trabajo por proyectos como la metodología que propicie su introducción definitiva. Bajo esta premisa, este estudio pretende identificar la presencia que contenidos históricos y patrimonio poseen en las aulas de Educación Infantil, y su relación con la implantación de la metodología por proyectos. Para ello, se ha desarrollado una investigación cuantitativa a través de un cuestionario. Esto ha permitido evaluar la situación al respecto sobre una muestra significativa de docentes de Educación Infantil de la Región de Murcia (España). Los resultados obtenidos aportan una inicial relación entre el desarrollo de la metodología por proyectos y la introducción de temáticas históricas, así como de la introducción del patrimonio como recurso, aunque el uso de este sigue siendo ocasional y poco ligado a los recursos que ofrece el propio entorno del alumnado.
\end{abstract}

PALABRAS ClAVE: Didáctica de la Historia, Educacion Patrimonial, Aprendizaje basado en objetos, investigación evaluativa, profesorado.

\section{PROJECT BASED LEARNING AS AN OPPORTUNITY TO INTRODUCE HISTORY AND HERITAGE AT EARLY YEARS-FOUNDATION STAGE}

\footnotetext{
ABSTRACT: Current trends in teaching and learning research encourage teachers to introduce historical contents at early years education, so as to promote

1. Trabajo realizado en el marco del Proyecto "La evaluación de las competencias y el desarrollo de capacidades cognitivas sobre Historia en Educación Secundaria Obligatoria" (EDU2015-65621-C3-2-R), financiado por el Ministerio de Economía y Competitividad de España / FEDER.
} 
the emerging development of the so called historical thinking skills. Cultural Heritage is shown as a key source in that process, and Project Based Learning $[\mathrm{PBL}]$ as an appropriate methodology to foster their final implementation. Under this assumption, this research aims to identify the presence of historical contents and heritage elements at Early Years Foundation Stage, and the connection with the use of PBL. To reach that goal, it has been developed a quantitative analysis based on a questionnaire, which has been completed by a representative sample of Early Years teachers of Región de Murcia (Spain). The results obtained lead us to confirm the relationship between the development of PBL and the introduction of historical contents, as well as in the use of heritage as a teaching source. However, it is still occasionally used and it is scarcely linked with the children's own environment.

KEYWORDS: History Education, Heritage Education, Evidence based practice, evaluative research, teachers.

Recibido: 02/12/2016

Aceptado: 20/07/2017

Correspondencia: Alejandro Egea Vivancos, Universidad de Murcia, Calle Campus Universitario, 12, 30100 Murcia. Email: alexegea@um.es.

\section{Introducción. El patrimonio cultural e historia en las aulas de EdUCACIÓN INFANTIL}

La riqueza que el patrimonio cultural aporta a las sociedades es tan innegable como la fragilidad que este posee y la amenaza que siempre planea sobre él. Su conservación depende en gran medida de la valoración que la sociedad haga del mismo y para ello es clave la acción educativa. Siendo conscientes de este último aspecto, es necesario abordar un estudio que muestre la presencia que este patrimonio posee en los distintos niveles educativos y así poder realizar un diagnóstico al respecto.

Es fundamental comenzar el análisis por la etapa de Educación Infantil, momento en el que se asientan las bases de los muchos aprendizajes que irán configurando la propia personalidad del niño y definiendo su rol como ciudadanos. En este sentido, la introducción de la Educación Patrimonial en estos primeros años se muestra de gran trascendencia al poder generar esos primeros vínculos con los testimonios de las muchas culturas que han poblado, pueblan y poblarán el entorno de los alumnos, preparándoles de este modo para la sociedad multiétnica y multirracial en la que viven (Wood y Holden, 1995). Además, la vinculación que el patrimonio cultural posee con el devenir histórico hace que este, en cualquiera de sus formas, sea considerado como un recurso idóneo para la historia al ser todas ellas huellas del pasado (Fines y Nichol, 1997; Luc, 1981).

Sin embargo, su inclusión en la enseñanza suele quedar estrechamente ligada a la existencia o no de contenidos históricos a impartir. Es éste precisamente el princi- 
pal problema que existe en Educación Infantil, donde la introducción de contenidos históricos queda a merced del deseo del docente, al no estar explícitamente incorporado al currículum educativo (Real Decreto 1630/2006), aunque puede ser asociado transversalmente a diversos bloques de contenidos (Miralles y Rivero, 2012). De la misma manera, la inclusión del patrimonio es residual y prácticamente se circunscribe al patrimonio inmaterial, a excepción de la mención a las producciones culturales de grupos sociales cercanos señalada en el objetivo 3 del área de "Conocimiento del entorno" (Real Decreto 1630/2006).

\section{La enseñanza de la historia en Educación Infantil}

Aún así, en la actualidad, la presencia de temáticas históricas es cada vez más frecuente en esta etapa (Castilla, 2005; Cooper, 2002; Gil y Rivero, 2014; Pérez, Baeza y Miralles, 2008). Y es que la introducción de metodologías alternativas y la incorporación de ciertas innovaciones en los métodos de enseñanza, está favoreciendo el desarrollo de propuestas de corte histórico por la gran variedad temática que aporta y las posibilidades que posee para el desarrollo de un trabajo interdisciplinar o globalizado (Harnett y Whitehouse, 2013).

Pero, además, la introducción de la historia en el sistema educativo permite dotar al alumnado de instrumentos de análisis, comprensión e interpretación que contribuyen a la formación integral (intelectual, social y afectiva) del alumnado (Prats, 2001; Santisteban, 2010). Pero, para conseguir esto, es necesario dejar de lado la concepción tradicional de la historia basada en el predominio de lo conceptual (Liceras, 1997). Ello implica abordar la disciplina como una forma de conocimiento con una metodología propia y que, por tanto, posibilita la comprensión del pasado a través de sus pruebas, conjugando lo conceptual con contenidos procedimentales propios de la labor del historiador, lo que se viene definiendo como pensamiento histórico (Lévesque, 2008; Seixas y Morton, 2013; Wineburg, 2001). Esta renovación de la disciplina está, paulatinamente, Ilegando a la primera etapa educativa (Cooper, 2013), con la intención de potenciar desde edades tempranas un conocimiento procedimental de carácter histórico basado en una primera familiarización de aspectos relacionados con la comprensión de la temporalidad (Chacón, 2009), la introducción de ciertas metodologías de indagación (Cooper, 2002) o el desarrollo de recursos y estrategias narrativas (Egan, 1991; Farmer y Heeley, 2004).

No obstante, no se puede obviar que la comprensión temporal entraña una gran dificultad para el alumnado de estas edades. La comprensión de estas primeras nociones temporales han estado durante largo tiempo ligadas a la necesidad de que el alumnado comprendiera y asimilara determinados elementos asociados al tiempo físico y cronológico (Hoodless, 1996). Es debido a su inicial vinculación con estas construcciones complejas que, tradicionalmente, se ha considerado la enseñanza del tiempo histórico fuera del alcance del alumnado más joven. En este sentido, las investigaciones psicoevolutivas Ilevadas a cabo por Piaget sobre el aprendizaje del tiempo en la infancia, dejaban a un lado la incorporación de contenidos históricos en el aula ya que según él su conocimiento del entorno (físico y temporal) que no 
fuera inmediato quedaba limitado a etapas superiores (Piaget, 1978). Sin embargo, las tendencias actuales se alejan de esta visión tan restrictiva del modelo piagetiano (Aranda, 2016; Dean, 1995; De Groot-Reuvekamp, Van Boxtel, Ros y Harnett, 2014; Hoodless, 1996; Levstik, 1993; Trepat, 2011; Woodhouse, 2001). Bajo esta misma perspectiva, otros autores también justificaban su presencia bajo la premisa de que el alumnado estaba vinculado al pasado a través de los medios de comunicación, la familia y el patrimonio cultural, entre otros (Wood y Holden, 1995) lo que les ofrece una imagen que puede ser poco precisa. De tal forma que, a día de hoy, los obstáculos que se presentan a la hora de vincular el conocimiento histórico se deben centrar más en las estrategias, recursos y metodología empleados que en las propias capacidades cognitivas del alumnado.

\section{El patrimonio cultural como recurso en Educación Infantil}

El patrimonio cultural se muestra como un recurso clave para favorecer la introducción de estas habilidades de pensamiento en edades tempranas (Cuenca y Domínguez, 2000; Luc, 1981; Pluckrose, 1993) y los museos se convierten en elementos imprescindibles para el tratamiento didáctico de la historia (Cuenca, 2011; Feliu y Hernández, 2011; Prats, 2001; Zabala y Roura, 2006). Son los museos y centros de interpretación los que pueden servir de plataforma para introducir lo patrimonial en las aulas a través del uso de las múltiples y variadas fuentes materiales que en ellos están depositadas. Estas permiten una interacción absoluta de un público variado, al no ser necesario contar con habilidades de lecto-escritura para afrontar su análisis (Crawford, 2016; Harnett y Whitehouse, 2013; Vella, 2010).

La introducción del trabajo con este tipo de fuentes posibilita la incipiente promoción de las habilidades propias del investigador y el desarrollo del pensamiento histórico en Educación Infantil a través del establecimiento de inferencias y deducciones en torno a las mismas, mediante el desarrollo de actividades que promuevan el análisis, la reflexión y la comunicación para comenzar a distinguir entre el pasado y el presente del entorno más cercano (Cooper, 2002; Hoodless, 1996). Estas propuestas pueden, por ejemplo, basarse en un modelo de preguntas en el que el alumnado se enfrenta, por vez primera, a la búsqueda de determinadas respuestas a través de su propia observación (Arias, Casanova, Egea, García y Morales, 2016) a partir de hechos u objetos significativos para el alumnado (Hales, 2015; Levstik y Barton, 2011; Santisteban y Pagès, 2011).

Sin embargo, pese a las posibilidades didácticas que este posee, su presencia en las aulas de Educación Infantil se muestra en principio escasa (Cuenca y Domínguez, 2000; Loison, 2008). En realidad, ya sea en el ámbito español o internacional, aún son limitadas las investigaciones que puedan corroborar o no esta afirmación. Es por ello por lo que, precisamente, la presente investigación adquiere gran relevancia, al aportar datos que permiten completar el panorama al respecto.

\section{El aprendizaje por proyectos como una metodología posibilitadora del cambio}

Sin duda alguna, las metodologías activas, como el aprendizaje basado en proyectos $(\mathrm{ABP})$, permiten convertir las aulas en espacios para la investigación y 
recreación de los hechos históricos y, por tanto, introducir el conocimiento histórico en edades tempranas (Miralles y Rivero, 2012). Esta metodología permite otorgar al alumnado un papel activo en el proceso de enseñanza-aprendizaje, y construir el conocimiento desde la investigación y relación de los saberes que conforman el sistema educativo (Hernández, 2000).

Desde el origen del concepto "proyecto" a finales del siglo XIX, fundamentado en las ideas de Dewey y su teoría del "aprender haciendo", o la acuñación del término actual en 1918 de manos de Kilpatrick (Pozuelos, 2007), el ABP actualmente supone "el protagonismo del estudiante en su proceso de enseñanza" (Hernández, 1996, p. 48), a través de la organización de ciertos contenidos curriculares "bajo un enfoque globalizador y significativo" (Muñoz y Díaz, 2009, p. 106), muchas veces basado en la experimentación y la generación de un aprendizaje más funcional sustentado en pruebas. Se aleja de la perspectiva de conocimientos fragmentados y alejados de los problemas próximos al entorno del alumnado, para abordar el aprendizaje desde una perspectiva transdisciplinar y un currículum en proceso de construcción (Hernández, 2000). Todo ello requiere, claro está, de un enfoque cooperativo, que conlleve la creación de un clima de interacciones basado en el compromiso y relaciones equilibradas por parte de los agentes implicados, fomentando a su vez, la creación de un entorno democrático. La actividad o lo motriz, la creatividad expresiva, la introducción del contexto familiar y la inclusión, son algunos de los fundamentos que caracterizarían a este modelo (Galeana, 2007; García, 2013; Pozuelos, 2007).

Las posibilidades didácticas que proporciona la metodología por proyectos hacen que, progresivamente, esta adquiera un papel más significativo en las aulas de Educación Infantil, aunque con matizaciones. En este sentido, el propio carácter interdisciplinar de la historia parece que está propiciando su mayor protagonismo en el desarrollo de estos proyectos de trabajo al aportar temáticas que generan un gran interés y a partir de las cuales se pueden articular múltiples interacciones conectadas con diversos campos de conocimiento (Harnett y Whitehouse, 2013). Teniendo en cuenta lo anterior, resulta interesante conocer hasta qué punto se está haciendo uso de la metodología por proyectos en esta etapa y su asociación con el trabajo de la disciplina histórica y la introducción de los elementos patrimoniales con el fin de establecer la relación entre ambos aspectos, a lo que intentaremos dar respuesta con esta investigación.

\section{Método}

\section{Objetivos}

Esta investigación responde a una serie de objetivos. Por un lado, (1) analizar el uso de la metodología por proyectos en el proceso de enseñanza-aprendizaje en Educación Infantil desde la perspectiva de los docentes, en segundo lugar, (2) valorar la presencia de contenidos históricos en dicha etapa, así como su relación con el método por proyectos y, finalmente, (3) describir la metodología, estrategias y recursos asociados al tratamiento del patrimonio cultural en Educación Infantil y su asociación al aprendizaje basado en proyectos. 


\section{Diseño de la investigación}

Para dar respuesta a estos objetivos se procedió a diseñar una investigación diagnóstica de corte cuantitativo que tuviera como población los docentes de Educación Infantil de la Región de Murcia. Gracias a un instrumento de evaluación propiamente cuantitativo, un cuestionario estructurado y escalas de valoración, se pretendía poder explicar, predecir y controlar el fenómeno objeto de estudio.

\section{Contexto y participantes}

El contexto elegido son los docentes de Educación Infantil de la Región de Murcia. Para la elección de los participantes se ha procedido a efectuar un muestreo no probabilístico intencional, que pretendía obtener sujetos que aportaran diferentes perspectivas. Esto permitiría contrastar las percepciones y experiencias en relación a la utilización de la metodología por proyectos y el tratamiento del patrimonio en este nivel. Con este fin se procuró contar con docentes que desarrollaran su labor educativa en dicha etapa en centros de titularidad pública y concertada así como que proporcionaran una variada muestra en cuanto a años de experiencia docente y a su formación académica.

Del total de la muestra $(\mathrm{N}=60), 41$ eran docentes de la escuela pública $(68,3 \%)$ y 19 de centros concertados (31,7\%). Atendiendo al género, casi la totalidad eran mujeres $(98,3 \%, n=59)$, reflejo perfecto de la realidad laboral actual regional (CREM, 2013-2014). Predominan aquellas que poseían en exclusiva la especialidad en Educación Infantil (95\%), siendo minoritarias las que habían cursado otras especialidades (Primaria, 3,3\%; Pedagogía, 1,7\%). En cuanto a la experiencia, la muestra se repartía de manera equitativa entre las diferentes franjas de edad.

\section{Recogida de información: instrumentos y procedimiento}

El instrumento seleccionado ha sido un cuestionario estructurado dirigido a los docentes de Educación Infantil, centrado en las siguientes categorías: frecuencia de uso y elaboración de la metodología por proyectos, presencia de la historia y el patrimonio cultural, y recursos y estrategias empleados para abordar contenidos históricos en esta etapa. La fase de validación se realizó mediante un juicio por expertos teniendo en cuenta aspectos como la presentación, instrucciones y estructura del cuestionario (Sánchez, Serrano y Alfageme, 2011), siendo igualmente analizado el grado de concordancia de sus valoraciones. Para ello, en primer lugar, se analizó el coeficiente o índice de concordancia $W$ de Kendall. Esta prueba facilitó valores referidos al grado de asociación entre cada una de las evaluaciones realizadas por los jueces. Esta prueba no paramétrica reveló un valor $W=.603, g l=3$ y $p=.000$, lo que mostró que existía concordancia, aunque a un nivel moderado (Siegel y Castella, 2005). Por su parte, la fiabilidad del cuestionario quedó establecida con una media de concordancia entre los expertos superior a 3.4 (Sánchez, Serrano y Alfageme, 2011). Del mismo modo, para conocer la consistencia interna de los ítems de escala Likert presentes en el cuestionario se utilizó el coeficiente Alfa de Cronbach, que arrojó un valor =.586. A pesar de que 
el indicador del cuestionario posee una consistencia interna moderada (George y Mallery, 2003), resulta aceptable para estudios exploratorios en las primeras fases de la investigación (Nunnally, 1967).

Finalmente, atendiendo a las puntuaciones y a las propuestas de mejora planteadas, se llevó a cabo la elaboración definitiva del instrumento de recogida de información (Tabla 1). El cuestionario resultante constaba de un total de 18 ítems: 4 de identificación (ítems 1-4), 11 de valoración en una escala Likert de 4 opciones, cuya escala oscila entre nunca (1) y siempre (4) (ítems 5-7, 9, 11, 13, 15-17) y 5 ítems de respuesta múltiple de carácter cerrado (ítems $8,10,12,14$ y 18) (Tabla 1).

Tabla 1. Items del cuestionario. Se excluyen aquellos correspondientes a los datos de identificación

\begin{tabular}{|c|c|}
\hline Ítem & Enunciado \\
\hline 5 & En la actualidad, el método empleado en el aula corresponde a una metodología por proyectos. \\
\hline 6 & Los proyectos son diseñados por el docente. \\
\hline 7 & Los proyectos son programados de forma colaborativa entre el equipo docente. \\
\hline 8 & En el caso de que los materiales empleados correspondan a una editorial, ¿cuál es el nombre? \\
\hline 9 & $\begin{array}{l}\text { ¿Se recogen contenidos relacionados con la disciplina histórica en la programación docente de } \\
\text { Educación Infantil? }\end{array}$ \\
\hline 10 & $\begin{array}{l}\text { ¿Con cuáles de estos contenidos generales han trabajado en los últimos cinco años? Puede } \\
\text { señalar más de una opción: }\end{array}$ \\
\hline 11 & Los Proyectos o unidades de tipo histórico están relacionados con el patrimonio cultural. \\
\hline 12 & ¿De qué manera se incorpora el patrimonio cultural en estos proyectos o unidades de trabajo? \\
\hline 13 & $\begin{array}{l}\text { En el caso de emplear la visita a los museos con los alumnos de Educación Infantil, ¿con qué } \\
\text { frecuencia se utiliza este recurso? }\end{array}$ \\
\hline 14 & ¿Qué tipo de museos han visitado? \\
\hline 15 & $\begin{array}{l}\text { Para la visita a los espacios museísticos se realizan actividades previas y finales de reflexión en } \\
\text { el aula. }\end{array}$ \\
\hline 16 & $\begin{array}{l}\text { En el caso de emplear la observación y manipulación de recreaciones de objetos del pasado } \\
\text { con el alumnado de Educación Infantil, ¿con qué frecuencia se utiliza este recurso? }\end{array}$ \\
\hline 17 & $\begin{array}{l}\text { En el caso de emplear los itinerarios didácticos (por el entorno) con el alumnado de Educación } \\
\text { Infantil, ¿con qué frecuencia se utiliza este recurso? }\end{array}$ \\
\hline 18 & $\begin{array}{l}\text { ¿Utiliza o ha utilizado alguna metodología diferente a esta, para trabajar el patrimonio cultural } \\
\text { en Educación Infantil? }\end{array}$ \\
\hline
\end{tabular}

Fuente: Elaboración propia.

\section{Procedimiento de la investigación}

Tras la confección, validación y modificación del cuestionario, se procedió a la fase de aplicación. Una vez cumplimentados los cuestionarios se definieron las variables y se conformó la matriz de datos a través del programa IBM SPSS Statistics for Windows (ver. 19). Se procedió a un análisis descriptivo univariable, consistente en el cálculo de estadísticos descriptivos, frecuencias y porcentajes para cada una de las variables ordinales y nominales. 


\section{Resultados}

Objetivo 1. Analizar el uso de la metodología por proyectos en el proceso de enseñanza-aprendizaje en Educación Infantil desde la perspectiva de los docentes.

Una vez analizados los ítems 5-8 se observa que un 15\% ( $n=9)$ del profesorado encuestado afirma emplear esta metodología "siempre", un 21,7\% ( $n=13)$ "con frecuencia", un $36,6 \%(n=22)$ "a veces" y un $26,7 \%(n=16)$ no lo emplea nunca. Su aplicación se hace de manera coordinada ya que un 31,8\% coordina sus acciones "con frecuencia" y un $47,7 \%$ declara hacerlo "siempre". De la misma manera, se muestra habitual la creación propia de los proyectos de trabajo pues un $34,1 \%$ afirman diseñarlos ellos mismos "con frecuencia" mientras que un $27,3 \%$ lo hacen "siempre". El diseño de materiales por parte de los docentes es más frecuente entre aquellos que incorporan de manera habitual en el aula el $\mathrm{ABP}$, ya que si se analizan los que afirman trabajar por proyectos y se descartan aquellos que dicen que no lo hacen o que lo hacen raras veces, se comprueba que el $54,5 \%$ diseña estos proyectos "con frecuencia" y un 13,6\% los diseña "siempre".

Los docentes que no elaboran sus propios materiales hacen uso de materiales curriculares elaborados por las editoriales. Estos suelen corresponderse con libros de texto compuestos por fichas de trabajo y lecturas complementarias asociadas a la temática seleccionada por la editorial. Atendiendo precisamente a las editoriales que más frecuentemente son utilizadas se observa que, entre los que afirman trabajar con proyectos, la editorial Santillana es la más empleada con un $52,3 \%(n=23)$, seguida de Edelvives por un $31,8 \%(n=14)$, siendo mucho más residuales otras editoriales. Sin embargo, entre los que afirman no utilizar los proyectos de trabajo, es Edelvives la más habitual con un $56,3 \%(n=9)$, seguida de Santillana con un $37,5 \%(n=6)$. Estos aspectos son de gran relevancia al definir en gran medida los contenidos a tratar y gran parte de las estrategias y recursos a utilizar en las aulas.

\section{Objetivo 2. Valorar la presencia de contenidos históricos en Educación Infantil.}

Ya sea o no asociados a proyectos, el análisis del ítem 9 refleja que la totalidad de la muestra ha incorporado alguna vez temáticas históricas en la programación docente de su aula, aunque el peso de su presencia en las programaciones diseñadas varía. Estos contenidos son integrados "siempre" por un $10 \%$ de los maestros, siendo éste el valor menos representativo. Por el contrario, la cifra se eleva hasta el $70 \%$ entre los que incluyen "a veces" contenidos relacionados con la disciplina histórica en su programación. Si se enfrentan los datos entre los que trabajan con proyectos y los que no, se comprueba que la introducción de contenidos históricos en las aulas está estrechamente relacionada al ABP (Tabla 2).

En relación a qué temáticas históricas se tratan en el aula, se observa cómo la más empleada es la relativa a los "Castillos" $(66,7 \%, n=40)$ y a continuación las que versan sobre "Museos" (53,3\%, $\mathrm{n}=32)$. Otros temas habituales son la "Prehistoria" $(46,7 \%, n=28)$ o "Los Egipcios" (26,7\%, n=16), temáticas que son coincidentes con las ofertadas por las editoriales señaladas. En un quinto grupo se incluyen propuestas añadidas por los docentes: temáticas variadas como "Pintores" o "Pintores locales" $(7 \%, n=7)$, los "Monumentos de la localidad" o "Historia local" $(10 \%, n=6)$, la "Vuelta al mundo" $(5 \%, \mathrm{n}=3)$ o, incluso, los "Dinosaurios" $(5 \%, \mathrm{n}=3)$ al entender algunos docentes esta temática como propia de la disciplina histórica. 
Tabla 2. Docentes que trabajan o no por proyectos y su relación con la incorporación de contenidos históricos en las aulas de Educación Infantil

\begin{tabular}{|c|c|c|c|c|}
\hline \multirow{2}{*}{$\begin{array}{c}\text { Trabaja con } \\
\text { proyectos }\end{array}$} & \multicolumn{2}{|c|}{ Siempre/Con frecuencia } & \multicolumn{2}{c|}{ A veces/Nunca } \\
\cline { 2 - 5 } & \multicolumn{2}{|c|}{$22(36,7 \%)$} & \multicolumn{2}{c|}{$38(63,3 \%)$} \\
\hline $\begin{array}{c}\text { Incluye contenidos } \\
\text { históricos }\end{array}$ & $\begin{array}{c}\text { Siempre/Con } \\
\text { frecuencia }\end{array}$ & A veces/Nunca & $\begin{array}{c}\text { Siempre/Con } \\
\text { frecuencia }\end{array}$ & A veces/Nunca \\
\cline { 2 - 5 } & $14(63,6 \%)$ & $8(36,4 \%)$ & $4(10,5 \%)$ & $34(89,5 \%)$ \\
\hline
\end{tabular}

Fuente: Elaboración propia.

\section{Objetivo 3. Describir la relación entre la metodología, estrategias y recursos asociados al tratamiento del patrimonio cultural en la etapa de Educación Infantil.}

Para dar respuesta a este objetivo, se preguntó a los docentes si los materiales empleados en el aula, ya fueran proyectos o unidades, versaban o no sobre el patrimonio cultural. En este sentido, se observa que, aunque todos afirman haberlo incluido alguna vez, la frecuencia es bastante desigual. Solo aquellos docentes que afirmaron hacer un uso habitual del ABP señalaron la presencia del patrimonio como una constante en el aula (Tabla 3).

Tabla 3. Frecuencia de la presencia o introducción del patrimonio cultural en Educación Infantil, y su relación con el empleo del aprendizaje basado en problemas

\begin{tabular}{|l|c|c|c|c|}
\hline & Nunca & A veces & $\begin{array}{c}\text { Con } \\
\text { frecuencia }\end{array}$ & Siempre \\
\hline Docentes en general & - & 54,7 & 40,6 & 4,7 \\
\hline Docentes que emplean ABP de manera esporádica & - & 50 & 45,5 & 4,5 \\
\hline Docentes que emplean ABP de manera habitual & - & 20,8 & 4,2 & 75 \\
\hline Docentes que elaboran sus propios proyectos & - & 45,2 & 51,6 & 3,2 \\
\hline
\end{tabular}

Fuente: Elaboración propia.

A continuación, interesaba averiguar cómo se estaba incluyendo el patrimonio en las aulas. De los datos analizados (Tabla 4), se observa la absoluta prevalencia de las imágenes y vídeos, que representan un $91,7 \%$, sobre el resto de recursos destinados al desarrollo del patrimonio. En contraposición, una de las estrategias menos utilizadas por los docentes (18,3\%) son las denominadas "maletas didácticas" o reproducciones de objetos arqueológicos.

Tabla 4. Estadísticas descriptivas sobre los recursos empleados para abordar el patrimonio cultural

\begin{tabular}{|l|c|c|}
\hline \multicolumn{1}{|c|}{ Ítems } & Frecuencia & Porcentaje \\
\hline Salidas por la ciudad & 39 & 65 \\
\hline Visitas a museos y/o exposiciones & 48 & 80 \\
\hline Imágenes y vídeos & 55 & 91,7 \\
\hline Reproducciones o maletas didácticas & 11 & 18,3 \\
\hline
\end{tabular}

Fuente: Elaboración propia. 
Si se analizan únicamente aquellos recursos que proporcionan un contacto directo del alumnado con el recurso patrimonial empleado, podemos constatar cómo los museos se muestra como la opción preferente, ya que un $80 \%(n=48)$ de los docentes afirma realizar visitas a espacios culturales como museos. Sin embargo, es interesante observar cómo su uso es bastante esporádico, ya que la mayor frecuencia de las visitas a los museos se sitúa en "a veces" (70\%). Aún así, se vuelve a observar cierta divergencia en el uso del recurso atendiendo a la metodología docente utilizada, aumentando la frecuencia de las visitas a museos entre aquellos que hacen uso del ABP (Tabla 5).

Tabla 5. Porcentajes relacionados con el tratamiento didáctico de los espacios museísticos. Frecuencia de visita

\begin{tabular}{|l|c|c|c|c|}
\hline \multicolumn{1}{|c|}{ Ítem } & Nunca & A veces & $\begin{array}{c}\text { Con } \\
\text { frecuencia }\end{array}$ & Siempre \\
\hline Docentes que visitan los museos & 15 & 70 & 13,3 & 1,7 \\
\hline $\begin{array}{l}\text { Docentes que visitan los museos entre los que } \\
\text { NO utilizan los proyectos }\end{array}$ & 2,3 & 77,3 & 18,2 & 2,3 \\
\hline $\begin{array}{l}\text { Docentes que visitan los museos entre los que } \\
\text { Sí utilizan los proyectos }\end{array}$ & 4,2 & 33,3 & 33,3 & 29,2 \\
\hline
\end{tabular}

Fuente: Elaboración propia.

Similares resultados arroja el análisis en torno a la frecuencia de uso de objetos e itinerarios o salidas didácticas (Tabla 6). Al respecto, en relación a los itinerarios, pese a ser utilizados por un alto porcentaje, estos se incorporan como estrategia minoritaria.

Tabla 6. Porcentajes de la frecuencia de utilización salidas didácticas por el entorno en la práctica docente

\begin{tabular}{|l|c|c|c|c|}
\hline \multicolumn{1}{|c|}{ Ítem } & Nunca & A veces & $\begin{array}{c}\text { Con } \\
\text { frecuencia }\end{array}$ & Siempre \\
\hline $\begin{array}{l}\text { Docentes que realizan salidas didácticas por el } \\
\text { entorno }\end{array}$ & 30 & 53,3 & 15 & 1,7 \\
\hline $\begin{array}{l}\text { Docentes que realizan salidas didácticas por el } \\
\text { entorno entre los que NO utilizan los proyectos }\end{array}$ & 42,1 & 50 & 7,9 & - \\
\hline $\begin{array}{l}\text { Docentes que realizan salidas didácticas por el } \\
\text { entorno entre los que Sí utilizan los proyectos }\end{array}$ & 9,1 & 59,1 & 27,3 & 4,5 \\
\hline
\end{tabular}

Fuente: Elaboración propia.

Otra cuestión interesante era averiguar qué tipos de museos se visitan con más frecuencia en esta etapa. A la vista de los resultados, los espacios más visitados corresponden a los museos asociados a las ciencias experimentales $(71,7 \%, n=43)$, seguidos de los museos de arte $(45 \%, \mathrm{n}=27)$ y los museos históricos o arqueológicos $(38,3 \%, n=23)$, siendo los menos visitados los museos etnográficos $(8,3 \%, n=5)$. 
En el caso en el que se llevaran a cabo estas visitas, se preguntó por la asiduidad con la que se planteaban actividades previas y posteriores a la visita para introducir y consolidar los contenidos que iban a ser trabajados en las mismas. En relación a esto, y una vez descartados aquellos que afirmaban no acudir a este tipo de espacios, un $43,1 \%$ y un $9,8 \%$ de la muestra afirman plantear su visita "siempre" y "con frecuencia", respectivamente, mediante actividades de contextualización para integrar adecuadamente los nuevos conocimientos adquiridos. Frente a esto, el 47,1\% "a veces" introduce esta secuencia.

\section{DisCUSIÓN E INTERPRETACIÓN DE LOS RESULTADOS}

La novedad de la investigación hace que se posean escasos elementos de comparación que permitan el cotejo de los resultados obtenidos. Como ya se ha dicho, mucho se ha señalado sobre la necesidad e importancia que historia y patrimonio poseen en esta etapa y la escasez de su presencia en el aula. Por ello, resultaba imprescindible abordar esta cuestión. En este sentido, los resultados mostrados permiten esbozar una introducción. En cuanto al primer objetivo de la investigación, en la actualidad y según el presente estudio, el ABP ha sido implementado alguna vez en las aulas de Educación Infantil por el 73,3\% del profesorado encuestado. Sin embargo, la periodicidad de la incorporación de proyectos de trabajo en el aula es poco frecuente y son pocos los que vertebran su práctica educativa de manera habitual en torno a los mismos. Cuando lo hacen, esta metodología se introduce mayoritariamente de manera coordinada entre los docentes de etapa y tienden a crear sus propios materiales para acompañar el desarrollo del proyecto. Aún así, más de un tercio de los encuestados utilizan como principal recurso el libro de texto, siendo Santillana y Edelvives las editoriales más utilizadas. Estas editoriales introducen temáticas asociadas a la historia como centro de interés de los proyectos diseñados. Así, entre ambas editoriales se cubren aspectos tales como la Prehistoria, los Egipcios, los Castillos (asociado a la Edad Media) o los Museos. Estos son coincidentes con aquellos que los docentes suelen introducir en sus aulas. Utilicen el material proporcionado por las editoriales, o no, estas parecen influir en las temáticas integradas en el aula. Entre los contenidos trabajados en las aulas, sorprende la alta frecuencia en el tratamiento de la Edad Media, a partir del análisis de la vida en los castillos (independientemente del pasado histórico y legado cultural de la zona geográfica de adscripción del centro). Sin embargo, aquellas temáticas directamente ligadas con el entorno del alumnado quedan relegadas a un plano totalmente secundario.

En la relación al segundo objetivo de esta investigación, centrado en valorar la presencia de contenidos históricos en las prácticas de enseñanza, los resultados obtenidos muestran que, en general, el profesorado introduce con escasa frecuencia contenidos relacionados con la disciplina histórica en las aulas. Por el contrario, la presencia de temáticas históricas parece mostrarse habitual como eje vertebrador del trabajo por proyectos. Por lo tanto, del análisis realizado parece desgajarse que la implementación del ABP va asociado a una mayor incorporación de contenidos históricos en el aula. Entre los que utilizan habitualmente este método, el 38,7\% afirma introducir "siempre" o "con frecuencia" contenidos de tipo histórico, mientras que aquellos que no hacen uso (o lo hacen esporádicamente) solo los incorporan "siempre" o "con frecuencia" un 6,2\%. 
Similar asociación se produce si se atiende al caso del uso del patrimonio cultural, dando respuesta así al tercer objetivo de la investigación. La incorporación de este recurso se establece con poca frecuencia, aunque la totalidad de los encuestados lo habían abordado en algún momento. De nuevo en este caso, el ABP parece favorecer su introducción en el aula, aunque únicamente cuando esta metodología aparece asociada al uso de libros de texto. Estos introducen frecuentemente entre sus fichas de trabajo y materiales complementarios imágenes diversas en relación a los contenidos a trabajar, teniendo el patrimonio un papel protagonista entre dichas imágenes. Estos soportes (fotografías e ilustraciones) son además los que los docentes afirman utilizar mayoritariamente en el caso de trabajar contenidos patrimoniales $(92,2 \%)$. Estos son seguidos de las visitas a museos $(80 \%)$ y las salidas al entorno a partir de itinerarios didácticos (65\%). Sin embargo, preguntados por la frecuencia en la que son integrados en la práctica educativa, se comprueba que ésta resulta altamente ocasional. Esta reflexión coincide con estudios previos en relación precisamente a la consideración e introducción del patrimonio en la etapa infantil en distintos contextos educativos (Cuenca y Domínguez, 2000; Loison, 2008). Dichos estudios situaban el patrimonio como un recurso residual en la práctica educativa en torno al que el docente, además, posee un escaso conocimiento y formación. Esto condiciona, precisamente, su introducción en el aula. De la misma manera, la falta de interacción entre docentes e instituciones patrimoniales así como la infrecuente elaboración de proyectos conjuntos entre la escuela y los espacios patrimoniales, determinan la gran distancia que se constata en esta investigación. Dicha distancia se amplía cuando se observa que, precisamente, los museos de tipo histórico, arqueológico y/o etnográfico son los menos visitados, por lo que la interacción con fuentes primarias de tipo objetual (de gran interés para esta etapa educativa), se ve reducida a la mínima expresión. Si, además, se tiene en cuenta que poco más de la mitad de los docentes dice preparar la visita con antelación a la misma y desarrollar actividades de consolidación tras su realización, se puede concluir que los museos son utilizados frecuentemente como espacios de ocio y no como fuentes de conocimiento o motivación para el desarrollo de actividades de indagación en torno al material expositivo. Solo si el docente consigue romper la barrera existente entre las piezas expuestas y los propios discentes se conseguirá convertir "objetos inanimados" en elementos repletos de información y historias asociadas (Fines y Nichol, 1997, p. 227).

\section{Conclusiones}

Antes de resumir los resultados de esta investigación, se ha de insistir en la necesidad de ampliar la investigación en este campo, no sólo en relación al tratamiento de la historia y la inclusión del patrimonio cultural en las aulas, sino también sobre su presencia en Educación Infantil (Barton y Levstik, 2008). Esta etapa ha quedado durante largo tiempo al margen de la investigación y son pocos los trabajos que analicen en profundidad su presencia en el aula, la acción educativa derivada de su aplicación y sus beneficios en el proceso de enseñanza-aprendizaje.

En este caso, se ha querido analizar la presencia que los contenidos históricos y los recursos patrimoniales poseían en esta etapa, y su relación con la creciente 
implementación de la metodología por proyectos. En este sentido, y tal y como se ha podido comprobar, el ABP favorece en principio la introducción de contenidos históricos en el aula de Educación Infantil, así como la integración del patrimonio para su desarrollo. Pero esta afirmación posee múltiples matizaciones, ya que esta primera investigación ha permitido comprobar cómo esta incorporación se ve igualmente ligada al uso o no de material editado, como son los libros de texto. De ahí que el uso e integración del patrimonio en las aulas se vea prácticamente limitado a recursos impresos que, por su parte, poco o nada tienen que ver con el entorno del alumnado al que van destinados. Cuando el profesorado elabora sus materiales no selecciona recursos patrimoniales para ilustrar los contenidos históricos, lo que lleva a detectar una escasa relación entre los docentes y la riqueza cultural del entorno. Esta desconexión se observa en el momento que estos señalan la escasa frecuencia con la que hacen uso de museos y exposiciones o el hecho de que apenas desarrollen itinerarios y visitas al propio patrimonio de la localidad o zonas aledañas. Pese a que los docentes tengan en cuenta este tipo de patrimonio en sus reflexiones, pocos son los que integran los mismos con cierta continuidad. De la misma manera, su inclusión no siempre conlleva su aprovechamiento didáctico, quedando en ocasiones la visita aislada respecto a la propia práctica educativa. Esto hace intuir la primacía del fin lúdico frente al educativo en las salidas programadas.

Pese a los beneficios que se observa que reporta el uso educativo del patrimonio, todavía no se ha conseguido una introducción efectiva del mismo. Son ya numerosas las propuestas al respecto que establecen pautas adaptadas a edades tempranas (Andreetti, 1993; Corbishley, 2011; Durbin, Morris y Wilkinson, 1990; Feliu y Jiménez, 2015; Hales, 2015; Smith y Holden, 1994; Santacana y Llonch, 2012; Egea, Pernas y Arias, 2014). Pero si se quiere llegar a las aulas, la formación del profesorado es clave $y$, en este proceso, las fuentes deben ser una estrategia a utilizar desde los primeros años de ingreso a la carrera (Montanares-Vargas y Llancavil-Llancavil, 2016). Igualmente, es imprescindible la colaboración entre museos y escuela para que estos sean utilizados como verdaderos lugares de aprendizaje, procurando un acercamiento a las fuentes primarias y al sentido de la investigación histórica. Si se quieren ampliar las muchas posibilidades que actualmente brindan los museos (Asensio y Pol, 2003), se ha de integrar su visita en una propuesta que conduzca a la reflexión, no solo a la observación.

En definitiva, el caso analizado sirve como espejo de la situación real española donde los contenidos históricos se suelen limitar a niveles superiores. Sin embargo, la inclusión del patrimonio y los métodos de indagación desde edades tempranas, favorecería ulteriormente una mejor comprensión y un mayor vínculo con la historia.

\section{REFERENCIAS BIBLIOGRÁFICAS}

Andreetti, K. (1993). Teaching History from Primary Evidence. London: David Fulton Publishers.

Aranda, A. M. (2016). Didáctica de las Ciencias Sociales en Educación Infantil. Madrid: Síntesis. 
Arias, L., Casanova, E., Egea, A., García, A. B. y Morales, M. J. (2016). Aprendiendo a tocar la historia. Las fuentes objetuales como recurso de aprendizaje en Educación Infantil y Primaria. En R. López-Facal (Ed.), Ciencias sociales, educación y futuro: Investigaciones en didáctica de las ciencias sociales (pp. 136-148). Santiago de Compostela: Red 14-Universidad de Santiago de Compostela.

Asensio, M. y Pol, E. (2003). Aprender en el museo. Íber, 36, 62-77.

Barton, K. C. y Levstik, L. S. (2008). 'Back when God was around and everything' Elementary children's understanding of historical time. En L. S. Levstik y K. C. Barton, Researching History Education. Theory, Method, and Context (pp. 71100). New York-London: Routledge.

Castilla, A. B. (2005). Arte e historia en el mundo infantil. Escuela Abierta: Revista de Investigación Educativa, 8, 231-267.

Chacón, A. (2009). Propuesta pedagógica y didáctica para la construcción del pensamiento histórico a partir del pensamiento narrativo en niños y niñas entre 5 y 7 años de edad. En O. Ibarra (Ed.), Investigación en educación, pedagogía y formación docente (pp. 231-239). Colombia: Fondo Editorial, UPN.

Cooper, H. (2002). History in the early years. London-New York: Routledge.

Cooper, H. (2013). Why must teaching and learning in history be creative? En R. Cooper (Coord.), Teaching history creatively (pp. 3-18). Oxon: Routledge.

Corbishley, M. (2011). Pinning down the past: archaeology, heritage, and education today. Woodbridge, Suffolk, UK; Rochester, NY: Boydell Press.

CREM (2013-2014). Profesorado según clase de enseñanza que imparte, titularidad y sexo, CREM y Servicio de Evaluación y Calidad Educativa. Estadística de la enseñanza no universitaria, Murcia. Recuperado de http://econet.carm.es/.

Cuenca, J. M. (2011). Concepciones del alumnado en Educación Infantil para la comprensión del medio sociocultural. Papel de las experiencias y el aprendizaje lúdico. En M. ${ }^{a}$ P. Rivero (Coord.), Didáctica de las Ciencias Sociales para Educación Infantil (pp. 111-129). Zaragoza: Mira Editores.

Cuenca, J. M. y Domínguez, C. (2000). Un planteamiento socio-histórico para educación infantil. El patrimonio como fuente para el trabajo de contenidos temporales. Íber, 23, 113-124.

Crawford, H. (2016). Using artefacts to develop young children's understanding of the past. Primary History, 72, 6-7.

Dean, J. (1995). Teaching History at Key Stage 2. Cambridge: Chris Kington.

De Groot-Reuvekamp, M. J., Van Boxtel, C., Ros, A. y Harnett, P. (2014). The understanding of historical time in the primary history curriculum in England and the Netherlands. Journal of Curriculum Studies, 46(4), 1-28. http://doi.org/10.108 0/00220272.2013.869837.

Durbin, G., Morris, S. y Wilkinson, S. (1990). Learning from objects: A teacher's guide. London: English Heritage.

Egan, K. (1991). La comprensión de la realidad en la educación infantil y primaria. Madrid: Ediciones Morata.

Egea, A., Pernas, S. y Arias, L. (2014). Re-construyendo la historia a partir del patrimonio arqueológico. En O. Fontal, A. Ibáñez Etxeberria y L. Martín Sánchez 
(Eds.), Reflexionar desde las experiencias. Una visión complementaria entre España, Francia y Brasil. Actas del II Congreso Internacional de Educación Patrimonial (pp. 1149-1157). Madrid: IPCE/OEPE.

Farmer, A. y Heeley, A. (2004). Moving between fantasy and reality: sustained, shared thinking about the past. En H. Cooper (Coord.), Exploring time and place through play. Foundation stage-key stage one (pp. 52-64). Oxon: David Fulton Publishers.

Feliu, M. y Hernández, F. X. (2011). 12 ideas clave. Enseñar y aprender historia. Barcelona: Graó.

Feliu, M. y Jiménez, L. (2015). Ciencias sociales y educación infantil (3-6): cuando despertó, el mundo estaba allí. Barcelona: Graó.

Fines, J. y Nichol, J. (1997). Teaching primary history. Oxford: Heinemann.

Galeana, L. (2007). Aprendizaje basado en proyectos. Universidad de Colima. Recuperado de http://ceupromed.ucol.mx/revista/PdfArt/1/27.pdf.

García, R. (2013). Enseñar y aprender en Educación Infantil a través de proyectos. Santander: Universidad de Cantabria.

George, D. y Mallery, P. (2003). Spss for Windows step by step: A Simple Guide and Reference. 11.0 Update (4. ${ }^{\mathrm{a}}$ ed.). Boston: Allyn \& Bacon.

Gil, J. y Rivero, P. (2014). Somos romanos. Análisis de una experiencia de trabajo por proyectos en Educación Infantil. Clio. History and History teaching, 40, 1-10. Recuperado de http://clio.rediris.es/n40/articulos/GilyRivero2014.pdf.

Hales, A. (2015). Learning in the early years through local people and places: developing historical concepts in the early years foundation stage. Primary History, 70, 6.

Harnett, P. y Whitehouse, S. (2013). Investigating Activities Using Sources. En H. Cooper (Ed.), Teaching History Creatively (pp. 29-50). London-New York: Routledge.

Hernández, F. (1996). Para comprender mejor la realidad. Cuadernos de Pedagogía, 243, 48-53.

Hernández, F. (2000). Los proyectos de trabajo: la necesidad de nuevas competencias para nuevas formas de racionalidad. Educar, 26, 39-51.

Hoodless, P. (1996). Children talking about the past. En N. Hall y J. Martello (Eds.), Listening to children think: exploring talk in the early years (pp. 100-116). London: Hodder \& Stoughton.

Lévesque, S. (2008). Thinking Historically. Toronto: UTP.

Levstik, L. S. (1993). Building a Sense of History in a First Grade Classroom. En J. E. Brophy (Ed.), Advances in Research on Teaching: Vol. 4. Research in Elementary Social Studies (pp. 1-31). Bingley: Emerald Group Publishing Limited.

Levstik, L. S. y Barton, K. C. (2011). Doing History: Investigating with Children in Elementary and Middle Schools. New York: Routledge.

Liceras, A. (1997). Dificultades en el aprendizaje de las ciencias sociales. Una perspectiva psicodidáctica. Granada: Grupo Editorial Universitario.

Loison, M. (2008). Obstáculos epistemológicos, didácticos y pedagógicos para una enseñanza articulada de patrimonio-historia. Íber, 57, 84-97.

Luc, J.-N. (1981). La enseñanza de la historia a través del medio. Madrid: Cincel. 
Miralles, P. y Rivero, P. (2012). Propuestas de innovación para la enseñanza de la historia en Educación Infantil. REIFOP, 15(1), 81-90. Recuperado de http://www. aufop.com/aufop/uploaded_files/revistas/133779829310.pdf.

Montanares-Vargas, E. y Llancavil-Llancavil, D. R. (2016). Uso de fuentes históricas en formación inicial de profesores. Magis. Revista Internacional de Investigación en Educación, 8(17), 85-98. http://doi.org/10.11144/Javeriana.m8-17.ufhf.

Muñoz, A. y Díaz, M. R. (2009). Metodología por proyectos en el área de conocimiento del medio. Revista Docencia e Investigación, 1(9), 101-126.

Nunnally, N. C. (1978). Psychometric Theory. New York: McGraw-Hill.

Pérez, E., Baeza, M. C. y Miralles, P. (2008). El rincón de los tiempos. Un palacio en el aula de Educación Infantil. Revista Iberoamericana de Educación, 48(1), 1-10. Recuperado de http://www.rieoei.org/expe/2627Egea-Maq.pdf.

Piaget, J. (1978). El desarrollo de la noción de tiempo en el niño (ed. orig. 1946). México: Fondo de Cultura Económica.

Pluckrose, H. (1993). Enseñanza y aprendizaje de la historia. Madrid: Morata.

Prats, J. (2001). Enseñar historia: Notas para una didáctica renovadora. Mérida: Junta de Extremadura. Recuperado de http://www.ub.edu/histodidactica/images/ documentos/pdf/ensenar_historia_notas_didactica_renovadora.pdf.

Pozuelos, F. J. (2007). Trabajo por proyectos en el aula: descripción, investigación y experiencias. Sevilla: Cooperación Educativa.

Real Decreto 1630/2006, de 29 de diciembre, por el que se establecen las enseñanzas mínimas del segundo ciclo de Educación infantil. (Boletín Oficial del Estado $n^{\circ} 4$, de 4 de Enero de 2007). Recuperado de https://www.boe.es/boe/dias/2007/01/04/ pdfs/A00474-00482.pdf.

Sánchez, P. A., Serrano, F. J. y Alfageme, M. B. (2011). Evaluación inter-jueces para el proceso de validación de un cuestionario para la investigación. Póster presentado en el XXII Simposio Internacional de Didáctica de las ciencias sociales: la evaluación en la didáctica de las ciencias sociales, Murcia, España.

Santacana, J. y Llonch, N. (2012). Manual de didáctica del objeto en el museo. Gijón: Trea.

Santisteban, A. (2010) La formación de competencias de pensamiento histórico. Clío \& Asociados, 14, 34-56. Recuperado de http://www.memoria.fahce.unlp.edu.ar/ art_revistas/pr.4019/pr.4019.pdf.

Santisteban, A. y Pagès, J. (Coords.) (2011). Didáctica del conocimiento del medio social y cultural en la Educación Primaria: ciencias sociales para comprender, pensar y actuar. Madrid: Síntesis.

Seixas, P. y Morton, T. (2013). The big six. Historical thinking concepts. Toronto: Nelson Education.

Siegel, S. y Castellan, N. J. (2005). Estadística no paramétrica aplicada a las ciencias de la conducta. Mexico D.F.: Editorial Trillas.

Smith, L., y Holden, C. (1994). 'I thought it was for picking bones out of soup...' Using artefacts in the primary school. Teaching History, 76, 6-9.

Trepat, C.-A. (2011). El apredizaje del tiempo en Educación Infantil. En M. ${ }^{\text {a }}$. Rivero (Coord.), Didáctica de las Ciencias Sociales para Educación Infantil (pp. 49-63). Zaragoza: Mira Editores. 
Vella, Y. (2010). Artefacts in history education. Teaching History, 54, 5.

Wineburg, S. (2001). Historical thinking and other unnatural acts. Philadelphia: Temple University Press.

Wood, L. y Holden, C. (1995). Teaching early years history. Cambridge: Chris Kington Pub.

Woodhouse, J. (2001) History in the foundation stage. Primary History, 27, 8.

Zabala, M. E. y Roura, I. (2006). Reflexiones teóricas sobre patrimonio, educación y museos. Revista de Teoría y Didáctica de las Ciencias Sociales, 11, 233-261. Recuperado de http://www.saber.ula.ve/bitstream/123456789/24036/2/articulo10.pdf. 